\title{
ZERO AND POLE DISTRIBUTION OF DIAGONAL PADÉ APPROXIMANTS TO THE EXPONENTIAL FUNCTION
}

\author{
Kathy A. Driver and Nico M. Temme \\ Dedicated to the memory of Douglas Sears
}

Abstract. The polynomials $P_{n}$ and $Q_{m}$ having degrees $n$ and $m$ respectively, with $P_{n}$ monic, that solve the approximation problem

$$
P_{n}(z) e^{-z}+Q_{m}(z)=\mathcal{O}\left(z^{n+m+1}\right)
$$

will be investigated for their asymptotic behaviour, in particular in connection with the distribution of their zeros. The symbol $\mathcal{O}$ means that the left-hand side should vanish at the origin at least to the order $n+m+1$. This problem is discussed in great detail in a series of papers by Saff and Varga. In the present paper we show how their results can be obtained by using uniform expansions of integrals in which Airy functions are the main approximants. We shall focus on the important diagonal case when $n=m$ and the polynomials $P_{n}$ and $Q_{n}$, as well as the remainder $E_{n, n}(z)=$ $P_{n}(z) e^{-z}+Q_{n}(z)$ can be expressed in terms of Hankel and Bessel functions. The approximate location of the zeros of $P_{n}, Q_{n}$ and $E_{n, n}$ are given in terms of the known zeros of certain Airy functions. An application is given in which the asymptotic information on the zeros is used to obtain an estimate in an approximation of the unit block function by means of the polynomials $P_{n}, Q_{n}$.

1991 Mathematics Subject Classification: 41A21, 30E15, 33C10, 30C15, 41A60.

1. Introduction. It is well known (cf. PERRON (1950, page 433)) that the solution of the Padé approximation problem for the exponential function, namely,

$$
P_{n}(z) e^{-z}+Q_{m}(z)=\mathcal{O}\left(z^{n+m+1}\right) \quad \text { as } \quad z \rightarrow 0,
$$

* Key words and phrases: Padé polynomials, asymptotic behaviour, uniform asymptotic methods, exponential function, Bessel functions, Airy functions, zero and pole distribution. 
where $P_{n}$ and $Q_{m}$ are polynomials of degree $n$ and $m$ respectively, with $P_{n}$ monic, is given by

$$
\begin{aligned}
P_{n}(z) & =\frac{1}{m !} \int_{0}^{\infty} t^{m}(t+z)^{n} e^{-t} d t, \\
Q_{m}(z) & =\frac{-1}{m !} \int_{0}^{\infty} t^{n}(t-z)^{m} e^{-t} d t .
\end{aligned}
$$

Explicit forms are (cf. PERRON (1950, page 436))

$$
\begin{gathered}
P_{n}(z)=n ! \sum_{k=0}^{n}\left(\begin{array}{c}
m+n-k \\
m
\end{array}\right) \frac{z^{k}}{k !}, \\
Q_{m}(z)=-n ! \sum_{k=0}^{m}\left(\begin{array}{c}
m+n-k \\
n
\end{array}\right) \frac{(-z)^{k}}{k !} .
\end{gathered}
$$

Let the remainder $E_{n, m}$ be defined by

$$
E_{n, m}(z)=P_{n}(z) e^{-z}+Q_{m}(z)
$$

then

$$
E_{n, m}(z)=\frac{(-1)^{m} z^{m+n+1}}{m !} \int_{0}^{1} u^{n}(1-u)^{m} e^{-u z} d u .
$$

The quantities $P_{n}, Q_{m}, E_{n, m}$ can be expressed in terms of (confluent) hypergeometric functions. We have

$$
\begin{aligned}
P_{n}(z) & =U(-n,-n-m, z)=z^{n+m+1} U(m+1, n+m+2, z) \\
Q_{m}(z) & =-\frac{n !}{m !} U(-m,-n-m,-z)=-\frac{n !}{m !}(-z)^{n+m+1} U(n+1, n+m+2,-z) \\
E_{n, m}(z) & =\frac{(-1)^{m+1} n ! z^{n+m+1}}{(n+m+1) !} M(n+1, n+m+2,-z) \\
& =\frac{(-1)^{m+1} n ! z^{n+m+1}}{(n+m+1) !} e^{-z} M(m+1, n+m+2, z) .
\end{aligned}
$$

In a sequence of papers Saff and Varga investigated the polynomials $P_{n}, Q_{m}$ and the remainder $E_{n, m}$, and the distribution of their zeros, for large values of $n, m$ with fixed ratio $\sigma=m / n$ (the final paper appeared in 1978). They used saddle point methods for the integrals defining the $U$ - and $M$-functions and found curves in the complex $z$-plane along which the zeros are cumulating. For $m=0$ their results agree with the earlier results obtained by Szegö on the distribution of the zeros of the exponential polynomial

$$
e_{n}(z)=\sum_{k=0}^{n} \frac{z^{k}}{k !}
$$

In this paper, we adopt a new approach for locating the zeros of $P_{n}, Q_{m}$ and $E_{n, m}$ by using uniform asymptotic approximations for these functions in terms of 
Airy functions. Approximate values of the zeros of $P_{n}, Q_{m}$ and $E_{n, m}$ are given in terms of the (known) zeros of certain Airy functions. For all positive integers $m$ and $n$, contour integral representations of $P_{n}, Q_{m}$ and $E_{n, m}$ can be given and uniform asymptotic methods applied to these contour integrals (cf. DRIVER \& TEMME (1997 and 1998)). Our investigations here will focus on the diagonal case $n=m$ where certain simplifications occur. For $n=m$, the quantities $P_{n}, Q_{n}$ and $E_{n, n}$ can be expressed in terms of Hankel and Bessel functions which permit Airy-type approximations to be done via Sommerfeld contour integrals, which seem not to be available in the general non-diagonal case.

2. The relations with Bessel and Hankel functions. In the diagonal case $n=m$ we have using the representations in (1.5) (cf. also ABRAmowitz \& STEGUN (1964, pages 509, 510))

$$
\begin{gathered}
P_{n}(z)=\pi^{-\frac{1}{2}} z^{n+\frac{1}{2}} e^{z / 2} K_{n+\frac{1}{2}}(z / 2), \\
E_{n, n}(z)=\frac{1}{2}(-1)^{n+1} \pi^{\frac{1}{2}} z^{n+\frac{1}{2}} e^{-z / 2} I_{n+\frac{1}{2}}(z / 2) .
\end{gathered}
$$

It follows immediately from (1.2) and (1.3) that, when $m=n$,

$$
Q_{n}(z)=-P_{n}(-z) \text {. }
$$

Of course, since the $[m / n]$ Padé approximant for $e^{-z}$ is $-Q_{m}(z) / P_{n}(z)$, the zeros of $P_{n}(z)$ are the poles of this Padé approximant and the above symmetry of $P_{n}$ and $Q_{n}$ means that knowledge about the zeros of either one of the polynomials suffices for both. Nevertheless, for our discussion, it is better to write $P_{n}$ and $Q_{n}$ in terms of different Bessel functions.

We have given in (2.1) the relations with modified Bessel functions. For obtaining the asymptotic expansions it is better to write the functions in terms of ordinary Bessel and Hankel functions. We recall the expansions (cf. WATSON (1944, page (201) or TEMme (1996, page 239))

$$
\begin{aligned}
& H_{n+\frac{1}{2}}^{(1)}(z)=\sqrt{2 /(\pi z)} e^{-(n+1) \pi i / 2+i z} \sum_{m=0}^{n}(+i)^{m}\left(n+\frac{1}{2}, m\right)(2 z)^{-m}, \\
& H_{n+\frac{1}{2}}^{(2)}(z)=\sqrt{2 /(\pi z)} e^{+(n+1) \pi i / 2-i z} \sum_{m=0}^{n}(-i)^{m}\left(n+\frac{1}{2}, m\right)(2 z)^{-m},
\end{aligned}
$$

where Hankel's symbol $(\alpha, m)$ is defined by

$$
(\alpha, m)=\frac{\Gamma\left(\frac{1}{2}+\alpha+m\right)}{m ! \Gamma\left(\frac{1}{2}+\alpha-m\right)}, \quad m=0,1,2, \ldots .
$$

Since

$$
\left(n+\frac{1}{2}, m\right)=\frac{(n+m) !}{m !(n-m) !} \quad \text { for } \quad m=0,1, \ldots, n
$$


a simple comparison of (2.3) and (2.4) with (1.3) and (1.2) respectively yields the relations

$$
\begin{aligned}
P_{n}(2 i z) & =-i(2 z)^{n} \sqrt{\pi z / 2} e^{i z} H_{n+\frac{1}{2}}^{(2)}(z), \\
Q_{n}(2 i z) & =-i(2 z)^{n} \sqrt{\pi z / 2} e^{-i z} H_{n+\frac{1}{2}}^{(1)}(z), \\
E_{n, n}(2 i z) & =-i(2 z)^{n} \sqrt{2 \pi z} e^{-i z} J_{n+\frac{1}{2}}(z),
\end{aligned}
$$

where the third line in $(2.5)$ follows from the relation

$$
J_{\nu}(z)=\frac{1}{2}\left[H_{\nu}^{(1)}(z)+H_{\nu}^{(2)}(z)\right] .
$$

It is known where the zeros of these Bessel functions occur when $n$ is large. For $J_{n+1 / 2}(z)$ they are located in the interval $(n, \infty)$. For $E_{n, n}(z)$ this gives an infinite set of zeros along the positive imaginary axis, in the interval $(2 i n, i \infty)$; there is a conjugate set in $(-2 i n,-i \infty)$. The Hankel functions have zeros along curves in the complex plane which start also at $z=n$; see ABramowitz \& STEGUN (1964, page 373). For $P_{n}(z)$ this gives $n$ zeros in the half-plane $\Re z<0$ along an arc from $2 i n$ to $-2 i n$. The arc cuts the real positive axis at $z=-2 n a$, where $a=0.66274 \ldots$ The zeros of $Q_{n}(z)$ are located in the right half-plane, and they follow from $Q_{n}(z)=-P_{n}(-z)$.

We give asymptotic representations of $P_{n}, Q_{n}$ and $E_{n, n}$ for large values of $n$ in terms of Airy functions. In Olver (1974, Ch. 11. §10) Airy-type expansions of the Bessel functions $J_{\nu}(\nu z)$ and $H_{\nu}^{(j)}(\nu z)$ are considered, but in the present case we need similar representations for $J_{n+\frac{1}{2}}(n z)$ and $H_{n+\frac{1}{2}}^{(j)}(n z)$.

3. Airy-type approximations for Bessel and Hankel functions. We give the main steps for deriving Airy-type approximations for Bessel and Hankel functions by using the Sommerfeld contour integrals. Since uniform asymptotic approximations of $J_{\nu}(\nu z), H_{\nu}^{(1)}(\nu z)$ and $H_{\nu}^{(2)}(\nu z)$ can be found in OLver (1974, pages 423-425) we can use the formulas in (2.5) to write down the corresponding approximations for $P_{n}\left[2 i\left(n+\frac{1}{2}\right) z\right], Q_{n}\left[2 i\left(n+\frac{1}{2}\right) z\right]$ and $E_{n, n}\left[2 i\left(n+\frac{1}{2}\right) z\right]$ immediately. In Olver's book the results are derived by using the differential equation of the Bessel functions, and the results are shown to hold in large domains of complex parameters and are provided with error bounds for the remainders in the expansions.

Because in the non-diagonal case, and also in Hermite-Padé approximations to the exponential functions (both aspects are studied in our papers DRIVER \& Temme $(1997,1998)$ ) these powerful results and methods are not available, we give in this preparatory paper the standard procedure based on the Sommerfeld integrals. A similar procedure will be used in the more general cases on more complicated integrals. In the Hermite-Padé case differential equations are not known for the corresponding polynomials.

We have (Olver (1974, page 58))

$$
J_{\nu}(z)=\frac{1}{2 \pi i} \int_{\mathcal{C}} e^{z \sinh t-\nu t} d t
$$


where $\mathcal{C}$ is a contour starting at $\infty-\pi i$ and terminating at $\infty+\pi i$. It follows that

$$
J_{n+\frac{1}{2}}(n z)=\frac{1}{2 \pi i} \int_{\mathcal{C}} e^{n \phi(t)-\frac{1}{2} t} d t, \quad \phi(t)=z \sinh t-t .
$$

Solving the equation $\phi^{\prime}(t)=0$, gives the saddle points

$$
t^{ \pm}= \pm \tau, \quad \tau=\operatorname{arccosh} \frac{1}{z} .
$$

When $0<z<1$, these points are real, when $z>1$ they lie on the imaginary axis. In order to obtain a standard form which can be used for obtaining a uniform expansion that is valid when the saddle points are close together, or coincide, we use the cubic transformation

$$
\phi(t)=\frac{1}{3} \zeta^{3}-\eta \zeta+A,
$$

where the saddle points $t^{ \pm}$should correspond with the saddle points $\pm \sqrt{\eta}$ in the $\zeta$-plane. This transformation was introduced by Chester, Friedman \& URSELL (1957). They showed that the mapping is one-to-one and analytic for all $z$ in a neighborhood of $z=1$, which is a local result. In fact, it can be shown (cf. DRIVER \& TEMME (1997)) that the mapping is one-to-one and analytic in a domain containing the path of integration.

The cubic transformation gives, upon substituting $t=t^{ \pm}, \zeta= \pm \sqrt{\eta}$,

$$
\frac{2}{3} \eta^{3 / 2}=\tau-\tanh \tau, \quad A=0 .
$$

If $0<z<1$ then $\tau$ and $\eta$ are positive. We can also write for positive $z$ :

$$
\begin{aligned}
\frac{2}{3} \eta^{3 / 2} & =\operatorname{arctanh} \sqrt{1-z^{2}}-\sqrt{1-z^{2}}, \quad 0<z \leq 1, \\
\frac{2}{3}(-\eta)^{3 / 2} & =\sqrt{z^{2}-1}-\arctan \sqrt{z^{2}-1}, \quad z \geq 1,
\end{aligned}
$$

which gives a better insight in the relation between $\eta$ and $z$. We can expand the arctanh-function to obtain

$$
\frac{2}{3} \eta^{3 / 2}=\frac{1}{3}\left(1-z^{2}\right)^{3 / 2}+\frac{1}{5}\left(1-z^{2}\right)^{5 / 2}+\ldots,
$$

which gives

$$
\eta=2^{1 / 3}(1-z)[1+\mathcal{O}(1-z)], \quad z \rightarrow 1 .
$$

This defines the relation near $z=1$. For complex values of $z$ this relation should be used with analytic continuation to define which branch of the multi-valued function $\eta^{3 / 2}$ is used.

Using the cubic transformation we obtain

$$
J_{n+\frac{1}{2}}(n z)=\frac{1}{2 \pi i} \int_{\mathcal{C}} e^{n\left(\frac{1}{3} \zeta^{3}-\eta \zeta\right)} f(\zeta) d \zeta, \quad f(\zeta)=e^{-\frac{1}{2} t} \frac{d t}{d \zeta}
$$


where $\mathcal{C}$ is a contour running from $\infty \exp (-\pi i / 3)$ to $\infty \exp (\pi i / 3)$, through the saddle point at $\zeta=\sqrt{\eta}$. The first order Airy-type uniform approximation is obtained by replacing $f(\zeta)$ with

$$
\begin{aligned}
f_{0} & =\frac{1}{2}[f(\sqrt{\eta})+f(-\sqrt{\eta})] \\
& =\left.\cosh \frac{1}{2} \tau \frac{d t}{d \zeta}\right|_{\zeta=\sqrt{\eta}} \\
& =\sqrt{\frac{z+1}{2 z}}\left(\frac{4 \eta}{1-z^{2}}\right)^{1 / 4} .
\end{aligned}
$$

This gives (compare with Olver (1974, page 425) and Abramowitz \& Stegun (1964, page 368))

$$
J_{n+\frac{1}{2}}(n z) \sim n^{-1 / 3} f_{0} \mathrm{Ai}\left(\eta n^{2 / 3}\right), \quad n \rightarrow \infty
$$

and, by using the third line in (2.5),

$$
E_{n, n}(2 i z n) \sim-i \sqrt{\pi} n^{-1 / 3}(2 z n)^{n+1 / 2} e^{-i z n} f_{0} \mathrm{Ai}\left(\eta n^{2 / 3}\right) .
$$

In a similar way we obtain for the Hankel functions

$$
\begin{aligned}
H_{n+\frac{1}{2}}^{(1)}(n z) & =\frac{1}{\pi i} \int_{-\infty}^{\infty+\pi i} e^{n \phi(t)-\frac{1}{2} t} d t \\
& =\frac{1}{\pi i} \int_{\mathcal{C}^{(1)}} e^{n\left(\frac{1}{3} \zeta^{3}-\eta \zeta\right)} f(\zeta) d \zeta \\
& \sim-2 n^{-1 / 3} f_{0} e^{2 \pi i / 3} \mathrm{Ai}\left(\eta n^{2 / 3} e^{2 \pi i / 3}\right) \\
Q_{n}(2 i z n) & \sim i \sqrt{\pi} n^{-1 / 3}(2 z n)^{n+1 / 2} e^{-i z n} f_{0} e^{2 \pi i / 3} \mathrm{Ai}\left(\eta n^{2 / 3} e^{2 \pi i / 3}\right) \\
H_{n+\frac{1}{2}}^{(2)}(n z) & =\frac{-1}{\pi i} \int_{-\infty}^{\infty-\pi i} e^{n \phi(t)-\frac{1}{2} t} d t \\
& =\frac{-1}{\pi i} \int_{\mathcal{C}^{(-1)}} e^{n\left(\frac{1}{3} \zeta^{3}-\eta \zeta\right)} f(\zeta) d \zeta \\
& \sim-2 n^{-1 / 3} f_{0} e^{-2 \pi i / 3} \mathrm{Ai}\left(\eta n^{2 / 3} e^{-2 \pi i / 3}\right) \\
P_{n}(2 i z n) & \sim i \sqrt{\pi} n^{-1 / 3}(2 z n)^{n+1 / 2} e^{i z n} f_{0} e^{-2 \pi i / 3} \mathrm{Ai}\left(\eta n^{2 / 3} e^{-2 \pi i / 3}\right)
\end{aligned}
$$

where $\mathcal{C}^{(j)}$ are contours running from $-\infty$ to $\infty \exp (j \pi i / 3), j=-1,1$. The results for the Hankel functions yield the Bessel function result because of the relation in (2.6) and (cf. Olver (1974, page 414))

$$
e^{-2 \pi i / 3} \mathrm{Ai}\left(z e^{-2 \pi i / 3}\right)+e^{2 \pi i / 3} \mathrm{Ai}\left(z e^{2 \pi i / 3}\right)+\mathrm{Ai}(z)=0 .
$$


We can obtain more terms in the approximations given in (3.9) - (3.11) by using standard methods for obtaining uniform expansion of integrals (cf. WoNG (1989)). On the other hand, as remarked at the beginning of this section, detailed information on this type of uniform expansions can be obtained from OLver (1974).

4. Locating the zeros. Having available the asymptotic approximations in terms of the Airy functions we can obtain approximations of the locations of the zeros of $P_{n}, Q_{n}, E_{n, n}$. The only zeros of the Airy function $\operatorname{Ai}(z)$ occur along the negative $z$-axis, and there are infinitely many zeros (cf. Olver (1974, page 415)). When the argument of the Airy function in (3.9) is negative, we have $\eta=\exp ( \pm i \pi)$, and $\eta^{3 / 2}=\mp i$. Because the right-hand side of (3.4) is purely imaginary when $z>1$, we conclude that an infinite string of zeros of $E_{n, n}(2 i z n)$ occurs near the intervals $(-\infty,-1),(1, \infty)$ (in fact all zeros are real and inside these intervals). The zeros of the polynomials $P_{n}(2 i z n), Q_{n}(2 i z n)$ are located along curves in the $z$-plane that are defined by $\eta \exp (-2 \pi i / 3)<0, \eta \exp (+2 \pi i / 3)<0$, respectively, where, again, the relation between $\eta$ and $z$ is given in (3.4).

To obtain a first approximation of these zeros, we use (3.9)-(3.11). Let $a_{j}$ be the zeros of $\operatorname{Ai}(z)$ (we have $a_{1}=-2.3381 \ldots$; see Abramowitz \& STEGUn (1964, page 478$)$ for more values). Then the zeros $z_{s}$ of $P_{n}(2 i z n)$ follow from the single term estimate obtained from the final line in (3.11)

$$
\eta_{s} \sim a_{s} e^{2 \pi i / 3} n^{-2 / 3}, \quad n \rightarrow \infty,
$$

and by inverting the relation between $\eta$ and $z$ (cf. (3.4)). More precise asymptotic estimates follow from using more terms in the uniform asymptotic approximations in (3.9)-(3.11) (cf. Driver \& TEMme (1997)). The zeros of $P_{n}(2 i z n)$ near $z=1$ satisfy (cf. (3.5))

$$
z_{j} \sim 1-a_{j} 2^{-1 / 3} e^{2 \pi i / 3} n^{-2 / 3}, \quad n \rightarrow \infty
$$

4.1. The condition for the zeros. We compare the condition for the location of the zeros of the quantities $P_{n}, Q_{n}, E_{n, n}$ as given in SAFF \& VARGA (1978) with the condition that follows from the uniform Airy-type asymptotic approximation. Saff and Varga introduce the quantity

$$
w_{1}(z)=\frac{2 i z e^{\sqrt{1-z^{2}}}}{1+\sqrt{1-z^{2}}},
$$

where we translated their notation into ours (that is, we take $\sigma=m / n=1$ and change $z$ into $i z$ ). According to Saff and Varga, the zeros of the three quantities $P_{n}, Q_{m}, E_{n, m}$ occur along curves in the $z$-plane defined by

$$
\left|w_{1}(z)\right|=1 \text {. }
$$

By using $z=1 / \cosh \tau$ (cf. (3.1)), a straightforward computation shows that

$$
\ln w_{1}(z)=\frac{1}{2} \pi i+\tanh \tau-\tau=\frac{1}{2} \pi i-\frac{2}{3} \eta^{3 / 2},
$$


where $\frac{2}{3} \eta^{3 / 2}$ is defined in (3.3). The condition (4.3) can be read as: $\ln w_{1}(z)$ is purely imaginary. So an equivalent formulation of (4.3) reads: $-\eta^{3 / 2}$ is purely imaginary, that is, the phase of $\eta$ equals $\pm \pi$ or $\pm \pi / 3$. In other words, $\eta$ is located on the rays where the zeros of $\operatorname{Ai}(z)$ and $\operatorname{Ai}\left(e^{ \pm 2 \pi / 3} z\right)$ lie. This is in agreement with our description of the location of the zeros given earlier in this section.

5. An application: approximating the block function. As an application we consider the approximation of the block function

$$
f(t)= \begin{cases}1, & \text { if } 0<x<1 \\ 0, & \text { otherwise }\end{cases}
$$

This function plays an important role in, for instance, the theory of electronics. We describe a method to obtain approximations of the block function by using Padé approximants of the exponential function. By using the estimates of the zeros of the polynomials $P_{n}(z)$ given in section 4 we derive an asymptotic estimate of the error in the approximation of the block function.

By taking the Laplace transform of $f$ and inverting we can write

$$
f(t)=\frac{1}{2 \pi i} \int_{\mathcal{L}} e^{s t}\left(1-e^{-s}\right) \frac{d s}{s}
$$

where $\mathcal{L}$ is a vertical line in the half-plane $\Re s>0$. To obtain smooth approximations of $f$ we can replace the exponential function $\exp (-s)$ with its Padé approximations, and we obtain

$$
f_{n}(t)=\frac{1}{2 \pi i} \int_{\mathcal{L}} e^{s t}\left[1+\frac{Q_{n}(s)}{P_{n}(s)}\right] \frac{d s}{s}, \quad n=1,2, \ldots
$$

For example,

$$
f_{1}(t)=2 e^{-2 t}, \quad f_{2}(t)=4 \sqrt{3} e^{-3 t} \sin (\sqrt{3} t) .
$$

From the results in the previous sections it follows that the polynomial $P_{n}(s)$ in (5.3) has $n$ simple zeros $s_{j}$ in the left half-plane $\Re s<0$. When we want to evaluate $f_{n}(t)$ of (5.3) numerically we can split up the rational function into partial fractions and evaluate the resulting integrals. By using residue calculus it is not difficult to prove that

$$
f_{n}(t)=\sum_{j=1}^{n} e^{s_{j} t} \frac{Q_{n}\left(s_{j}\right)}{s_{j} P_{n}^{\prime}\left(s_{j}\right)} .
$$

In this way we obtained the first elements given in (5.4) and in Figure 5.1. Observe that $s=0$ is not a pole in (5.3).

It is of interest to know the asymptotic nature of the representation in (5.5) for large values of $n$. When we order the zeros $s_{j}$ of $P_{n}(s)$ with respect to their real parts, a sum of residues as in (5.5) gives an excellent representation with respect 
Figure 5.1:

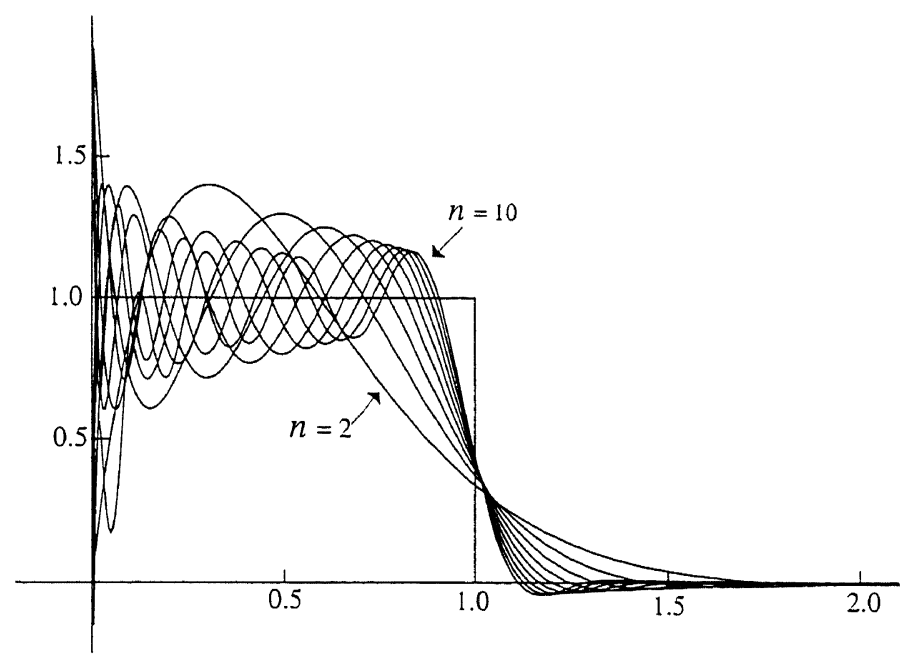

The functions $f_{n}(t)$ of (5.3) that approximate the block function $n=2,3, \ldots, 10$. The rate of approximation for large values of $n$ is described in (5.7), for $t>1$.

to large values of $t$. Surprisingly, the situation is different in the present case for large values of $n$.

The zeros $s_{j}$ with the largest real part (whose corresponding terms in (5.5) dominate the large $t$-behaviour) occur near $\pm 2 i n$. The behaviour of the $s_{j}$ near $s=2$ in follows from $(4.1)$ :

$$
s_{j} \sim 2 i n-a_{j} 2^{2 / 3} e^{7 \pi i / 6} n^{1 / 3}, \quad n \rightarrow \infty .
$$

Similar approximations hold near $s=-2 i n$, the real axis being an axis of symmetry. From (2.5) it follows that

$$
\frac{Q_{n}\left(s_{j}\right)}{s_{j} P_{n}^{\prime}\left(s_{j}\right)}=e^{-s_{j}} \frac{2 i H_{n+\frac{1}{2}}^{(1)}\left[s_{j} /(2 i)\right]}{s_{j} H_{n+\frac{1}{2}}^{(2)^{\prime}}\left[s_{j} /(2 i)\right]}=e^{-s_{j}} \mathcal{O}\left(n^{-2 / 3}\right),
$$

where we have used well-known estimates of the Hankel functions:

$$
H_{\nu}^{(1)}(\nu z)=\mathcal{O}\left(\nu^{-1 / 3}\right), \quad H_{\nu}^{(2)^{\prime}}(\nu z)=\mathcal{O}\left(\nu^{-2 / 3}\right), \quad \nu \rightarrow \infty
$$

with $z \sim 1$ (cf. Abramowitz \& Stegun (1964, pages 368-369)). Hence,

$$
f_{n}(t)=e^{(t-1) s_{1}} \mathcal{O}\left(n^{-2 / 3}\right)=\mathcal{O}\left[n^{-2 / 3} e^{-(t-1)\left|a_{1}\right| 2^{2 / 3} \cos (\pi / 6) n^{1 / 3}}\right],
$$

as $n \rightarrow \infty$ (with $a_{1}=-2.3381 \ldots$ ), which estimate holds if $t>1$. 
We see that the expected estimate $f_{n}(t) \sim 1$ for $0<t<1$ cannot be obtained in this way. Apparently, if $0<t<1$, all exponentially large contributions in (5.5) cancel against each other. When we concentrate on the error in the approximation, that is, on

$$
f(t)-f_{n}(t)=\frac{-1}{2 \pi i} \int_{\mathcal{L}} e^{s t} \frac{E_{n, n}(s)}{P_{n}(s)} \frac{d s}{s}, \quad n=1,2, \ldots,
$$

we can use saddle point methods. We can write this in the form

$$
f(t)-f_{n}(t)=\frac{(-1)^{n+1}}{2 i} \int_{\mathcal{L}} e^{2 w(t-1)} \frac{I_{n+\frac{1}{2}}(w)}{K_{n+\frac{1}{2}}(w)} \frac{d w}{w},
$$

where we used the modified Bessel function representations given in (2.1). We are not pursuing this further, because it is outside the scope of this paper.

We conclude this section by stating an interesting property of the approximations $f_{n}(t)$ defined in (5.3). We have

$$
\int_{0}^{\infty} f_{n}^{2}(t) d t=1, \quad n=1,2, \ldots
$$

which, on the one hand, says that the $L^{2}$-norm of the approximant is the same as that of the block function, but on the other hand, it means that the electronic system, based upon these functions $f_{n}(t)$, is stable.

To show (5.10) we use (5.5):

$$
I=\int_{0}^{\infty} f_{n}^{2}(t) d t=\sum_{j=1}^{n} \frac{Q_{n}\left(s_{j}\right)}{s_{j} P_{n}^{\prime}\left(s_{j}\right)} \int_{0}^{\infty} e^{s_{j} t} f_{n}(t) d t .
$$

We know from the earlier literature on Padé approximations to the exponential function (cf. SAFF \& VARGA (1978) and their earlier papers) that all zeros $s_{j}$ of $P_{n}(s)$ are in the left half-plane $\Re s<0$. Hence, inverting (5.3), we have

$$
I=-\sum_{j=1}^{n} \frac{Q_{n}\left(s_{j}\right)}{s_{j}^{2} P_{n}^{\prime}\left(s_{j}\right)}\left[1+\frac{Q_{n}\left(-s_{j}\right)}{P_{n}\left(-s_{j}\right)}\right]=-\sum_{j=1}^{n} \frac{Q_{n}\left(s_{j}\right)}{s_{j}^{2} P_{n}^{\prime}\left(s_{j}\right)},
$$

because $Q_{n}\left(-s_{j}\right)=-P_{n}\left(s_{j}\right)=0$. It is not difficult to verify that

$$
I=\frac{-1}{2 \pi i} \int_{\mathcal{C}}\left[1+\frac{Q_{n}(s)}{P_{n}(s)}\right] \frac{d s}{s^{2}}
$$

where $\mathcal{C}$ is a vertical line in the left half-plane such that all zeros of $P_{n}$ are at left of $\mathcal{C}$; on $\mathcal{C}$ we integrate from $-i \infty$ to $i \infty$. Using $1+Q_{n}(s) / P_{n}(s)=s+\mathcal{O}\left(s^{2}\right)$ as $s \rightarrow 0$ (which follows from the representations in (1.2) and (1.3) with $n=m$ ), we see that the integrand has a simple pole at the origin, with residue equal to 1 . We shift $\mathcal{C}$ across the pole at the origin, picking up the residue, and observe that the remaining integral over $\mathcal{C}$ in the right half-plane vanishes. Hence, $I=1$. 
6. Concluding remarks. We have shown how to obtain the location and asymptotic approximation of zeros of the polynomials that constitute the diagonal Padé approximations to the exponential function. By using uniform Airy-type asymptotic expansions it is possible to give a clear description of the distribution of the zeros and their asymptotic approximation. We have compared our method with Saff \& Varga's approach for locating the zeros of the polynomials.

We have used the asymptotic estimates of the zeros for obtaining an asymptotic estimate of functions that are smooth approximations to the block function, and we have proven a stability condition for these approximations.

In DRIVER \& TEMME (1997) more details are given on the non-diagonal case. Again we use Airy-type approximations to obtain the distribution of the zeros and the asymptotic estimates for the zeros of the polynomials.

\section{REFERENCES}

1. Abramowitz, M. And Stegun, I.A., Handbook of mathematical functions with formulas, graphs and mathematical tables, Nat. Bur. Standards Appl. Series 55, U.S. Government Printing Office, Washington, D.C., 1964.

2. Chester, C., Friedman, B. And Ursell, F., An extension of the method of steepest descent, Proc. Cambridge Philos. Soc. 53 (1957), 599-611.

3. Driver, K.A. and Temme, N.M., On polynomials related with Hermite-Padé approximations to the exponential function, J. of Approx. Theory 95 (1998), 101-122.

4. Asymptotics and zero distribution of Padé polynomials associated with the exponential function, J. of Comp. and Applied Math. 89 (1997), 97-114.

5. Olver, F.W.J., Asymptotics and special functions, Academic Press, New York, 1974.

6. Perron, O., Die Lehre von den Kettenbrüchen, Chelsea, New York, 1950.

7. SAFF, E.B. And VARGA, R.S., On the zeros and poles of Padé approximants to $e^{z}$. III, Numer. Math. 30 (1978), 241-266.

8. Temme, N.M., Special functions: An introduction to the classical functions of mathematical physics, Wiley, New York, 1996.

9. Watson, G.N., A treatise on the theory of Bessel functions, Cambridge University Press, London and New York, 1944.

10. Wong, R., Asymptotic approximations of integrals, Academic Press, New York, 1989.

Mathematics Department, University of the Witwatersrand, P.O. Wits 2050, South AFrica.

CWI, P.O. Box 94079, 1090 GB Amsterdam, The Netherlands.

EMAIL: 036kad@cosmos.wits.ac.za, nicot@cwi.nl

Received 21 November 1996 and in revised form 6 February 1997. 